\title{
The Use of Skin Effect for Measurement of Thickness of Sputtered Protective-Coatings
}

\author{
Josef Timmerberg, Jade Hochschule, Germany, Andris Martinovs, Rezekne Higher Education Institution, Latvia
}

\begin{abstract}
This paper deals with the development of a mathematical model that describes the skin effect in cylindrical conductors with protective coating. The model allows calculating the distribution of current density and magnetic field intensity, as well as determining electrical impedance, resistance, reactance and current in separate layers. It is established that electrical impedance at constant frequencies decreases linearly, if thickness of a protective coating grows. This fact can be used for electrical measurement of thickness of a protective coating.
\end{abstract}

Keywords - Measurement, skin effect, sputtered protectivecoating, thickness.

\section{INTRODUCTION}

The development and implementation of technologies of sputtered protective coatings require control over thickness of these coatings. One of the non-destructive and easily automatable methods that allows measuring thickness of an electrically conductive coating may be associated with the use of skin effect [1]. Let us use the skin effect theory (discussed in many literature sources, e.g., [2] - [6]) to determine in which situations this effect can be used for the measurement of thickness of a protective coating. In work [7] a calculation method of frequency-dependent resistance and internal inductance of a solid cylindrical conductor is developed. Skin effect reduces the volume of sample and changes its electrical resistance [8]. Modeling of skin effect for solving specific engineering problems is viewed in many authors' publications, for example, in [9] - [16].

The purpose of this paper is to develop a mathematical model that describes the skin effect in cylindrical conductors with a protective coating. It would allow assessing necessary conditions and developing a method for electrical measurement of thickness of a protective coating.

\section{MATHEMATICAL MODEL}

Conductive coating with thickness $\Delta$ is sputtered on a conductive cylindrical element with inside/ outside diameter $d_{1} / d_{2}$ (see Fig. 1).

High-frequency alternating current $I$ flows through a cylindrical element in the $z$-axis direction. In this situation skin effect is observed; high-frequency current flows only through a thin layer of cylindrical conductor's outside diameter. As $\Delta<<d_{2}$, the problem of skin effect can be solved for a one-dimensional case. Cylindrical conductor with sputtered protective coating will be changed to a rectangle conductor with height $\pi d_{2}$ and width $b$. Alternating current $I$ (in the $z$-axis direction) flows through it. There is no magnetic field in internal air space $\left(d<d_{1}\right)$ of cylindrical body, because $\oint \overrightarrow{\vec{H}} d \vec{s}=0$, therefore this space can be not viewed. As a basis for the development of this method, a calculation example from [2] will be used, where on surface $x=0$ the magnetic field intensity $H=0$. Thereby, it remains to observe only the area of conductor, which has coordinates $0 \leq x \leq a$ (let us mark it $\{i\}$ ) and the current conductive protective coating layer (e.g., TiN with $\mathrm{Al}$ ) with coordinates $a \leq x \leq b$ (let us mark it $\{m\}$ ). For these areas one dimensional skin equation is valid with directed vector potential directed along the $z$-axis $\vec{A}=\vec{e}_{z} A$.

$$
A_{i / m}=\frac{d^{2} A_{i / m}}{d x^{2}}=a_{i / m}^{2} A_{i / m}
$$
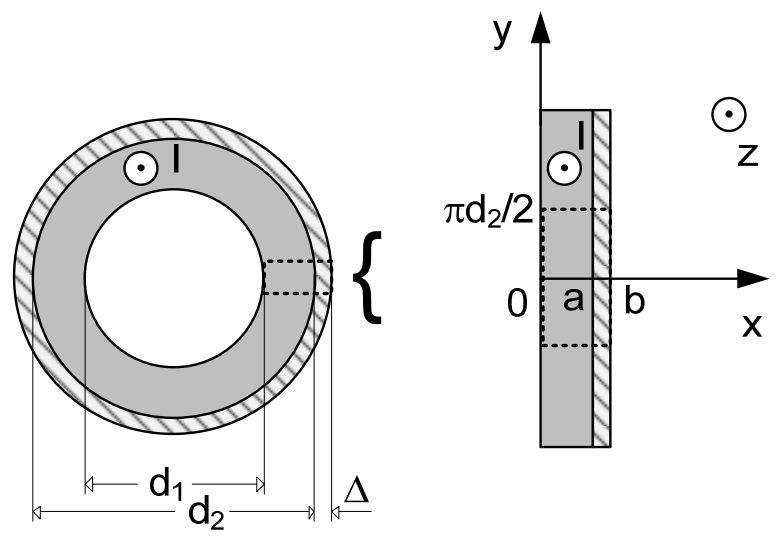

Fig. 1. Geometry of the model.

For the description of the field, vector potential $\vec{A}(x)$ with $\operatorname{rot}(\vec{A})=\vec{B}$ is used, where $\alpha=\sqrt{j \omega k \mu}=\frac{1+j}{\delta}-$ the skin constant, $\delta=\sqrt{\frac{2}{\omega k \mu}}-$ the skin depth, $\omega-$ the angular frequency of current, $k$-the specific electrical conductivity, $\mu$ - the absolute magnetic permeability, $j$ - the imaginary unit, $B$ - the magnetic flux density.

General solutions to (1) for areas $\{i\}$ and $\{m\}$ are searched as follows:

$$
\left\{\begin{array}{c}
A_{i}(x)=C_{i} \cosh \left(a_{i} x\right) \\
A_{m}(x)=C_{m} \cosh \left(a_{m} x\right)+D_{m} \sinh \left(a_{m} x\right)
\end{array}\right.
$$

In this case, the vector potential does not depend on coordinates $y$ and $z(d A / d y=d A / d z=0)$; current flows in the $z$-axis direction. It means that the magnetic field intensity has $y$-axis direction $\vec{H}(x)=\vec{e}_{y} H(x)$. Intensity can be calculated for areas $\{i\}$ and $\{m\}$ using (2) and well-known relevance of electrodynamics $\vec{B}=\mu \vec{e}_{y} H(x)=\operatorname{rot}\left(\vec{e}_{z} A\right)=-\vec{e}_{y} \frac{d A}{d x}$ : 


$$
\left\{\begin{array}{c}
H_{i}(x)=\frac{\alpha_{i}}{\mu_{i}} C_{i} \sinh \left(a_{i} x\right) \\
H_{m}(x)=\frac{\alpha_{m}}{\mu_{m}} C_{m} \sinh \left(a_{m} x\right)+\frac{\alpha_{m}}{\mu_{m}} D_{m} \cosh \left(a_{m} x\right)
\end{array}\right.
$$

Equation systems (2) and (3) have 3 unknown constants $C_{i}$, $C_{m}, D_{m}$. They can be determined using these conditions:

a) condition of continuity of vector potential $A_{i}(a)=A_{m}(a)$;

b) condition of continuity of magnetic field intensity $H_{i}(a)=H_{m}(a)$;

c) the magnetic field intensity vector integral by closed contour (see a dotted rectangle in Fig. 1) is equal to a sum of covered currents $\oint \vec{H} d \vec{s}=I$. After integration by this contour it is possible to obtain $H_{m}(b) \pi d_{2}=I$.

Using these conditions and (2) and (3) it is possible to obtain:

from a) and (2)

$C_{i} \cosh \left(a_{i} a\right)=C_{m} \cosh \left(a_{m} a\right)+D_{m} \sin \left(a_{m} a\right)$

from b) and (3)

$$
\frac{\alpha_{i}}{\mu_{i}} C_{i} \sinh \left(a_{i} a\right)=\frac{\alpha_{m}}{\mu_{m}} C_{m} \sinh \left(a_{m} a\right)+\frac{\alpha_{m}}{\mu_{m}} D_{m} \cosh \left(a_{m} a\right)
$$

from c) and (3)

$$
-\frac{\alpha_{m}}{\mu_{m}} C_{m} \sinh \left(a_{m} b\right)-\frac{\alpha_{m}}{\mu_{m}} D_{m} \cosh \left(a_{m} b\right) \pi d_{2}=I
$$

These equations are written in a matrix form

$$
\begin{aligned}
& \left(\begin{array}{ccc}
\cosh \left(a_{i} a\right) & -\cosh \left(a_{m} a\right) & -\sin \left(a_{m} a\right) \\
\frac{\alpha_{i}}{\mu_{i}} \sinh \left(a_{i} a\right) & -\frac{\alpha_{m}}{\mu_{m}} \sinh \left(a_{m} a\right) & -\frac{\alpha_{m}}{\mu_{m}} \cosh \left(a_{m} a\right) \\
0 & -\frac{\alpha_{m}}{\mu_{m}} \sinh \left(a_{m} b\right) & -\frac{\alpha_{m}}{\mu_{m}} \cosh \left(a_{m} b\right)
\end{array}\right) \\
& \cdot\left(\begin{array}{c}
C_{i} \\
C_{m} \\
D_{m}
\end{array}\right)=\left(\begin{array}{c}
o \\
0 \\
\frac{I}{\pi d_{2}}
\end{array}\right)
\end{aligned}
$$

Unknown constants $C_{i}, C_{m}$ and $D_{m}$ can be calculated numerically. Therefore vector potential (2) and magnetic field intensity (3) in both electrically conductive layers $\{i\}$ and $\{m\}$ are determined.

Alternating current density in layers $\{i\}$ and $\{m\}$ is calculated using equations of electrodynamics $\vec{J}=k \vec{E}$ and $\vec{E}=-j \omega \vec{A}$ (where $E-$ the electric field):

$$
\left\{\begin{array}{c}
J_{i}(x)=-j \omega k_{i} C_{i} \cosh \left(a_{i} x\right) \\
J_{m}(x)=-j \omega k_{m}\left(C_{m} \cosh \left(a_{m} x\right)+D_{m} \sinh \left(a_{m} x\right)\right.
\end{array}\right.
$$

At high frequencies, if calculations are performed throughout the thickness $\left(d_{2}-d_{1}\right) / 2$ of layer $\{i\}$, there are problems with numerical solving of (4). Therefore it is assumed:

if $\left(d_{2}-d_{1}\right) / 2>12 \delta_{i}$, then $a=12 \delta_{i}-$ the current in layer $\{i\}$ flows only near the external surface; if $\left(d_{2}-d_{1}\right) / 2 \leq 12 \delta_{i}$, then $a=\left(d_{2}-d_{1}\right) / 2$ - the current flows throughout the thickness of layer $\{i\}$.

Values of current density in (5) are complex numbers, which consist of real part $J_{R e}(x)$ and imaginary part $J_{I m}(x)$.

In practice, the root mean square (RMS) of current density $J B(x)$ is significant that can be calculated for layers $\{i\}$ and $\{m\}$ as follows:

$$
J B_{\frac{i}{m}}(x)=\frac{J_{\max }(x)}{\sqrt{2}}=\frac{\sqrt{J_{R e}(x)^{2}+J_{I m}(x)^{2}}}{\sqrt{2}},
$$

where $J_{\max }(x)$ - the AC current density amplitude at a point with coordinate $x$.

Knowing $J_{i}(x)$ and $J_{m}(x)$, it is possible to calculate currents, which flow in layers $\{i\}$ and $\{m\}$ :

$$
\left\{\begin{array}{c}
I_{i}=\frac{\pi d_{2}}{\sqrt{2}}=\left|\int_{0}^{a} J_{i}(x) d x\right| \\
I_{m}=\frac{\pi d_{2}}{\sqrt{2}}=\left|\int_{0}^{b} J_{m}(x) d x\right|
\end{array}\right.
$$

This equation system can be used for checking the numerical calculations. If $I_{i}+I_{m}=I$, then calculation is true.

By means of Poynting vector, it is possible to determine inside electrical impedance

$$
Z=-I^{2} \oiint_{A}(\vec{E} \times \vec{H}) d \vec{A}
$$

where $E$ - the electric field, $H$ - the magnetic field intensity on conductor's surface $A$.

As in the given situation, electric field vector $E$ is directed by the $z$-axis, magnetic field intensity vector $H$ is directed by the $y$-axis, as well as on the conductor internal surface $H(0)=0$ and on the conductor external surface $H(b) \neq 0$, then

$$
Z=\frac{\pi d_{2} l}{I^{2} k_{m}} J_{m}(b) H_{m}(b)
$$

where $l$ - the length of conductor. Impedance of layers $\{i\}$ and $\{m\}$ :

$$
\left\{\begin{array}{c}
Z_{i}=\frac{\pi d_{2} l}{I_{i}^{2} k_{i}} J_{i}(a) H_{i}(a) \\
Z_{m}=\frac{\pi d_{2} l}{I_{m}^{2} k_{m}} J_{m}(b) H_{m}(b)-J_{m}(a) H_{m}(a)
\end{array}\right.
$$

As both layers of conductor are connected in parallel circuits, then:

$$
\frac{1}{Z}=\frac{1}{Z_{i}}+\frac{1}{Z_{m}}
$$

$Z$ is a complex value, whose real part characterises resistance $R$, but imaginary part - reactance $X$.

\section{RESUlTS AND DisCUSSION}

Calculations for a steel tube of length $l=153 \mathrm{~mm}$ and inside/outside diameter $d_{1} / d_{2}=6 / 8 \mathrm{~mm}$ were performed using Mathcad software. A protective coating with thickness $\Delta$ is sputtered on the tube. In the calculations such material constants were used: specific electrical conductivity of steel 
$\kappa_{i}=(1,01 \pm 0,32) \cdot 10^{6} \mathrm{~S} / \mathrm{m}$; relative magnetic permeability of steel $\mu_{i}=27,9 \pm 2,0$; specific electrical conductivity of Al-TiN coating $\kappa_{m}=(0,43 \pm 0,11) \cdot 10^{6} \quad \mathrm{~S} / \mathrm{m}, \quad$ relative magnetic permeability $\mu_{m}=1$. [1]

An example for the distribution of current density $|J(x)| /$ $\sqrt{2}$ (RMS) in the tube with a protective coating is given in Fig. 2 and Fig. 3, where $|J(x)|$ - the amplitude of current density, coordinate $x$ is radially directed; the distribution of magnetic field intensity (amplitude) $H(x)$ is depicted - in Fig. 4 and Fig. 5; AC frequency $f=100 \mathrm{MHz}$; the total current in conductor $I=33,27 \mathrm{~mA}$; thickness of a protective coating $\Delta=1,6 \mu \mathrm{m}$. Units of measurement: $[x]=1 \mathrm{~m} ;[J(x)]=1 \mathrm{~A} / \mathrm{m}^{2} ;[H(x)]=1 \mathrm{~A} / \mathrm{m}$.

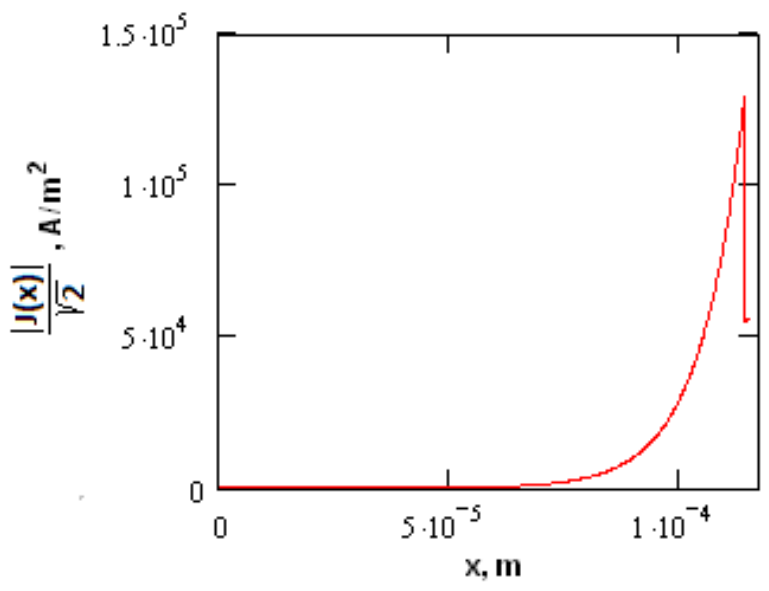

Fig. 2. Distribution of current density in the tube with a protective coating at $100 \mathrm{MHz}$.

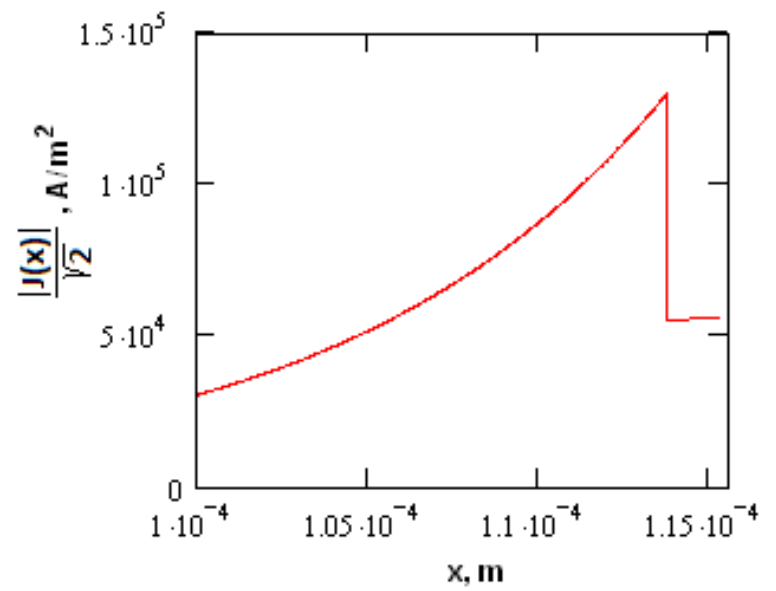

Fig. 3. Distribution of current density in the tube with a protective coating at $100 \mathrm{MHz}$ (expanded scale).

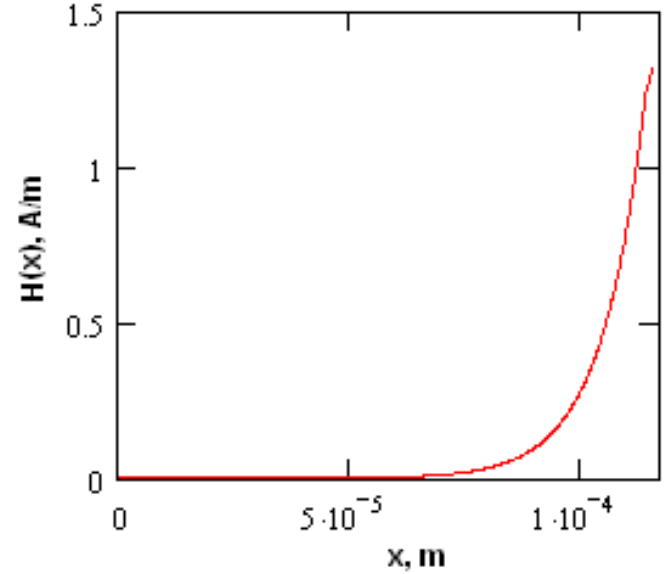

Fig. 4. Distribution of magnetic field intensity in the tube with a protective coating at $100 \mathrm{MHz}$.

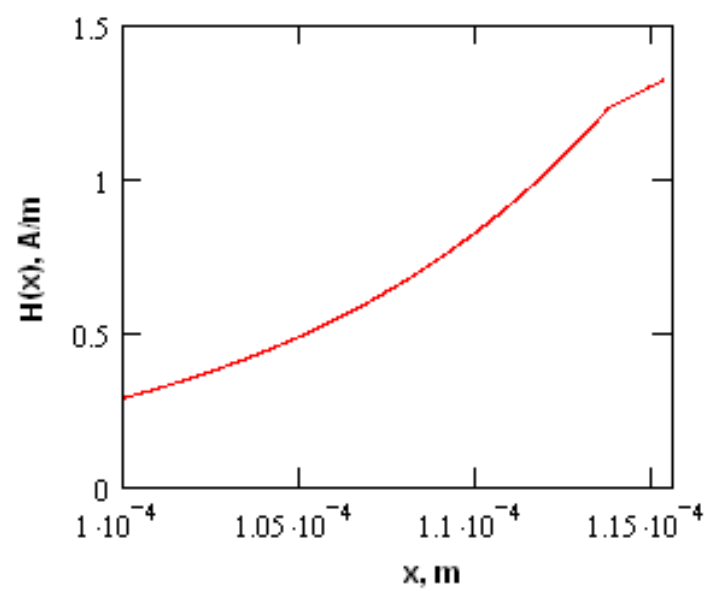

Fig. 5. Distribution of magnetic field intensity in the tube with a protective coating at $100 \mathrm{MHz}$ (expanded scale).

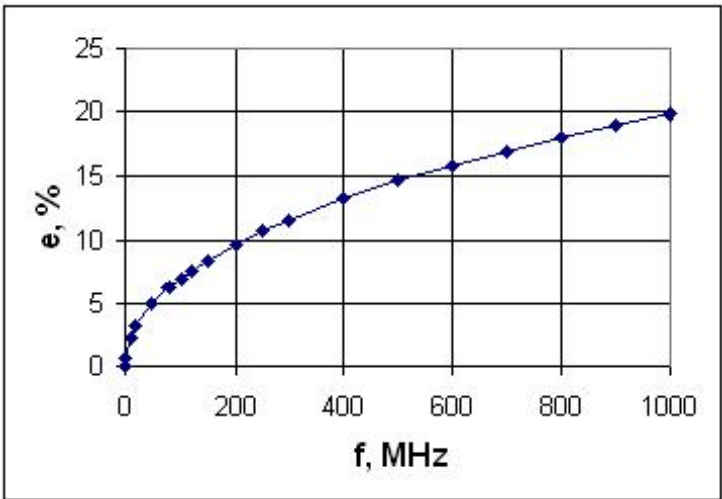

Fig. 6. Part of current that flows through a protective coating (thickness of 1.6 $\mu \mathrm{m})$ depending on $\mathrm{AC}$ frequency. 


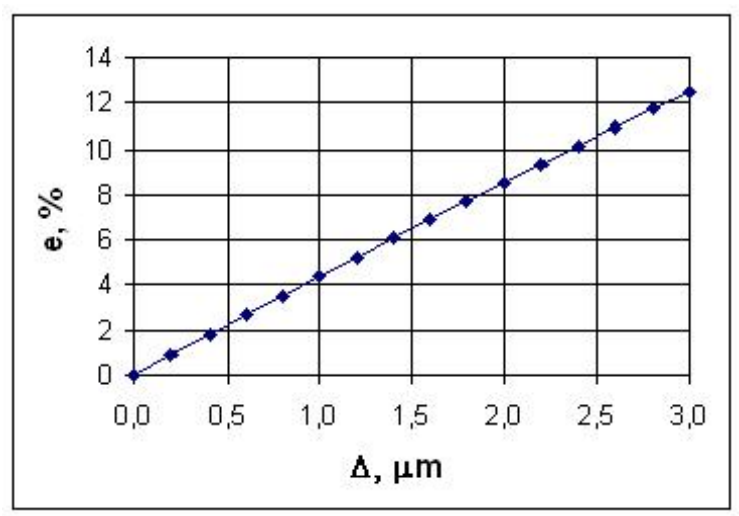

Fig. 7. Part of current that flows through a protective coating depending on the thickness of a layer at a frequency of $100 \mathrm{MHz}$.

If $f=1 \mathrm{MHz}$, then in calculations we obtain $Z=0.064+0.063 j$ $\Omega$. Experimentally measured resistance for a given sample $R=0.0643 \Omega$. [1]. Obviously, the numerical and experimental results fully coincide. It confirms the correctness of the given model.

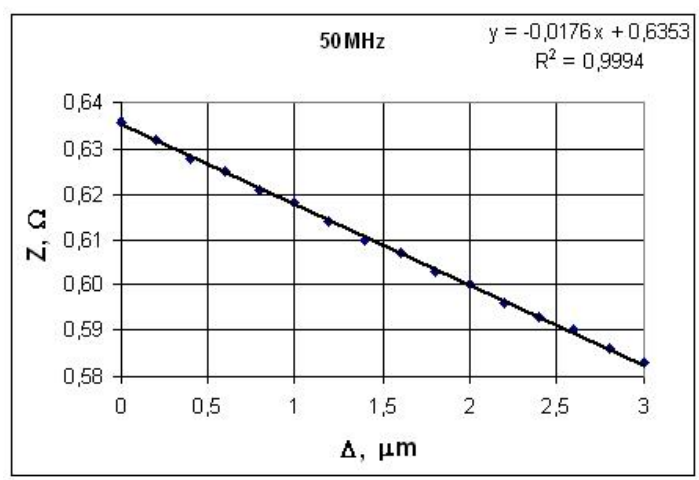

Fig. 8. Electrical impedance depending on the thickness of sputtered layer at $50 \mathrm{MHz}$.

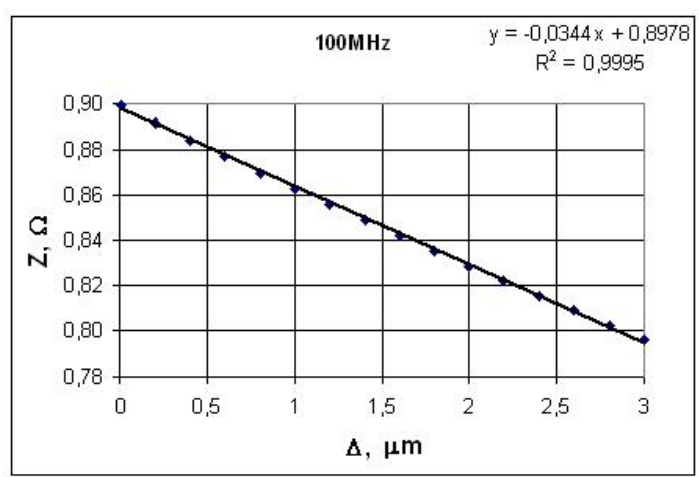

Fig. 9. Electrical impedance depending on the thickness of sputtered layer at $100 \mathrm{MHz}$.

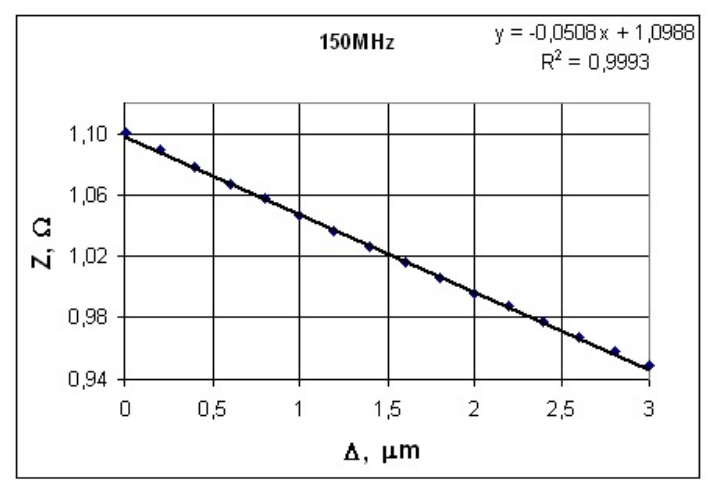

Fig. 10. Electrical impedance depending on the thickness of sputtered layer at $150 \mathrm{MHz}$.

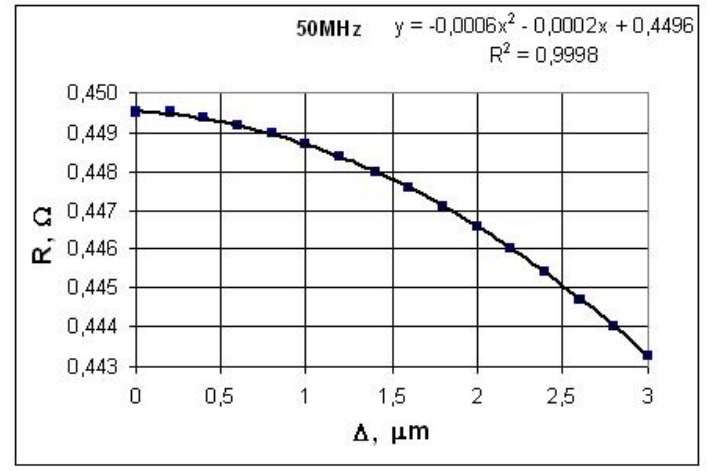

Fig. 11. Electrical resistance depending on the thickness of sputtered layer at $50 \mathrm{MHz}$.

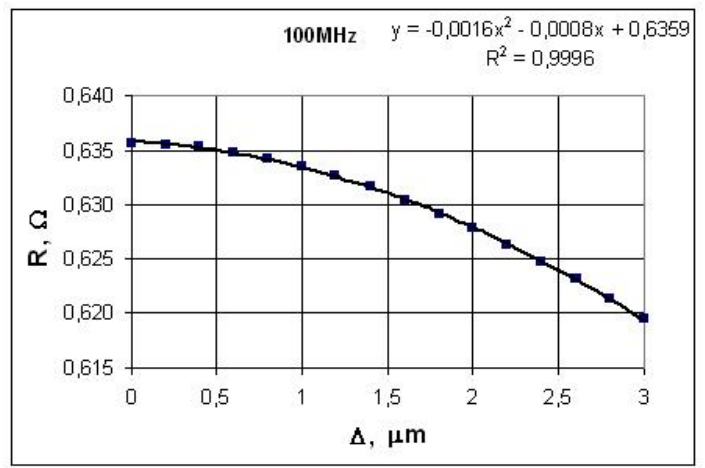

Fig. 12. Electrical resistance depending on thickness of sputtered layer at 100 MHz.

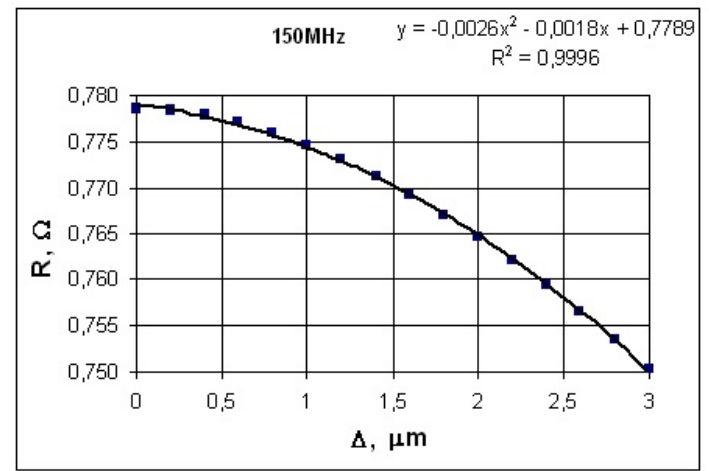

Fig. 13. Electrical resistance depending on the thickness of sputtered layer at $150 \mathrm{MHz}$. 
Let us define value $e$, which characterises part of current that flows in a protective coating:

$$
e=\frac{I_{m}}{I_{i}+I_{m}}
$$

Dependence of value $e$ on $\mathrm{AC}$ frequency at $\Delta=1,6 \mu \mathrm{m}$ is demonstrated in Fig. 6. Dependence of value $e$ on the thickness of a protective coating at $f=100 \mathrm{MHz}$ may be seen in Fig. 7 .

The higher value $e$, the larger part of current flows in a protective coating. To measure the thickness of a protective coating electrical gauges can be used with lower accuracy.

Figures 8-13 illustrate the dependence of electrical impedance $Z$ and resistance $R$ of the tube with a protective coating on the thickness of sputtered layer at AC frequency $f=50 \mathrm{MHz}, f=100 \mathrm{MHz}, f=150 \mathrm{MHz}$.

The higher AC frequency, the faster values $Z$ and $R$ change depending on the thickness of a protective coating. Consequently, the higher AC frequency, the lower accuracy of electrical gauges is necessary to measure the thickness of a protective coating.

If the thickness of a protective coating at a constant frequency grows, then electrical impedance $\mathrm{Z}$ linearly decreases.

\section{CONCLUSIONS}

Within the framework of the study a mathematical model has been developed that describes the skin effect in cylindrical conductors with a protective coating. The model can also be used for multilayer sputtered protective coatings - in this case, only the number of equations in systems (2) - (6), (10), (11) grows. The model allows calculating the distribution of current density and distribution of magnetic field intensity in cylindrical conductors with a protective coating, determining electrical impedance, resistance and reactance of separate layers, as well as setting current in each layer at any AC frequency and any thickness of a protective coating.

It is established that electrical impedance at constant frequencies decreases linearly, if the thickness of a protective coating grows. This means it is possible to use the skin effect for the measurement of thickness of a protective coating: at constant frequency it is necessary to measure the electrical impedance of cylindrical conductor without a protective coating and impedance of the same conductor with a protective coating, whose thickness of coating is known; then it is necessary to construct characteristic $Z=Z(\Delta)$ that in a given situation is a straight line; using this characteristic and measuring electrical impedance for similar conductors with sputtered protective coating, it is possible to determine the thickness of protective coating $\Delta$.

The model allows evaluating the necessary accuracy of electrical gauges (e.g., LCR meter, voltmeter, and ammeter) to measure thickness of a protective coating. Varying the thickness of a protective coating, changes in electrical parameters of cylindrical conductor become more quintessential, if $\mathrm{AC}$ with higher frequency is used; it means that at higher frequencies it is possible to use electrical gauges with lower accuracy for the measurement of thickness of a protective coating.

\section{ACKNOWLEDGMENTS}

This research has been supported by the Latvian Council of Science within the project "Development of Technology for the Creation of Multicomponent Nanostructured Protective Coatings for Industrial Products" No.10.0009.

\section{REFERENCES}

[1] A. Martinovs, J. Timmerberg, K. Savkovs, A. Urbahs and P.Beckmann, A method for determination of specific electrical resistance of steel and nano-coating sputtered on it: The 8th International Scientific and Practical Conference ,Environment. Technology. Resources”, vol.2, pp. 118-124, June 20-22, 2011, Rezekne, Latvia.

[2] A. Nethe and H.D. Stahlmann, Einfürung in die Feldtheorie. Berlin: Verlag Dr. Köster, 2003.

[3] L. Hannakam, Einfürung in die Feldtheorie. TU Berlin: VorlesungsNiederschrift, 1974.

[4] J. D. Jackson, Classical Electrodynamics. $3^{\text {rd }}$ edition. New York: John Wiley \& Sons, Inc., NY, 2004.

[5] K. Simonyi, Theoretische Elektrotechnik. Berlin: VEB Deutscher Verlag der Wissenschaften, 1989.

[6] V. Malo Machado, M. Eduarda Pedro, J. A. Brandao Faria and D.Van Dommelen, "Magnetic field analysis of three-conductor bundles in flat and triangular configurations with the inclusion of proximity and skin effects," Electric Power Systems Research, vol. 81, issue 11, pp 2005 2014, 2011 http://dx.doi.org/10.1016/j.epsr.2011.06.010

[7] O. M. O. Gatous and J. Pissolato, Frequency-dependent skin-effect formulation for resistance and internal inductance of a solid cylindrical conductor: IEE Proceedings - Microwaves, Antennas and Propagation, vol.151, issue 3, pp. 212-216, June 1, 2004 http://dx.doi.org/10.1049/ip-map:20040469

[8] R. J. Lopez, "Simultaneous observation of Barkhausen pulses and jumps in resistance fluctuations in ferromagnetic materials," Review of Scientific Instruments, vol. 70, number 1, pp. 171-174, January 1999. http://dx.doi.org/10.1063/1.1149560

[9] P. Rasilo, E. Dlala, K. Fonteyn, J. Pippuri, A. Belahcen and A. Arkkio, "Model of laminated ferromagnetic cores for loss prediction in electrical machines," IET Electric Power Applications, vol.5, issue 7, pp. 580-588, August 2011.

http://dx.doi.org/10.1049/iet-epa.2010.0270

[10] O. Bottauscio, M. Chiampi and D. Chiarabaglio, "Advanced model of laminated magnetic cores for two-dimensional field analysis" IEEE Transactions on Magnetics, vol. 36, issue 3, pp. 561-573, May 2000. http://dx.doi.org/10.1109/20.846219

[11] Oh Suk Kyung, "Accurate transient simulation of transmission lines with the skin effect," IEEE Transactions on Computer-Aided Design of Integrated Circuits and Systems, vol. 19, issue 3, pp. 389-396, March 2000. http://dx.doi.org/10.1109/43.833207

[12] P. K. Mahanta, P. and K. A. Rocky, Skin effect analysis for carbon nano material based interconnects at high frequency: International Conference on Informatics, Electronics \& Vision (ICIEV), pp. 1-6, May 17-18, 2013, Dhaka, Bangladesh.

[13] E. Abbaspour, R. Sarvari, A. Akbarzadeh and M. Rostami, Accurate numerical model for surface scattering, grain boundary scattering, and anomalous skin effect of copper wires: 2013 IEEE 5th International Nanoelectronics Conference (INEC), pp. 209-210, January 2-4, 2013, Singapore, Singapore

[14] M. D'Amore, M. S Sarto and A. G. D'Aloia, Skin-Effect Modelling of Carbon Nanotube Bundles. The High-Frequency Effective Impedance: IEEE International Symposium on Electromagnetic Compatibility (EMC), pp.847-852, July 25-30, 2010, Fort Lauderdale, USA.

[15] M. Magdowski, S. Kochetov and M. Leone, Modelling the skin effect in the time domain for the simulation of circuit interconnects: 2008 International Symposium on Electromagnetic Compatibility - EMC Europe, pp.1-6, 2008, Hamburg, Germany.

[16] Yu Du and W. Dai, "Capture high-frequency partial inductance more accurately by gauss quadrature integration with skin-effect model," IEEE Transactions on Microwave Theory and Techniques, vol. 54, issue 3, pp. 1287-1294, March 2006. http://dx.doi.org/10.1109/TMTT.2005.864096 


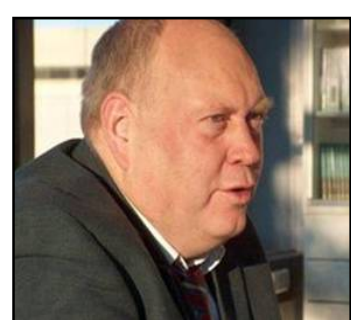

Jose Timmerberg holds a degree of Doctor of Engineering Sciences and is a Professor at the Jade University of Applied Sciences. Education: in 1981 - TU Berlin, Doctor in Electromagnetics; in 1978 - Dipl.-Ing. Electrical Engineering; in 1975 - the University of Applied Science Paderborn/Soest, Dipl.-Ing. (FH), Electrical Engineering, Technical University (TU) Berlin.

Work experience: research work (1981- 1983) and Director of Automation Engineering (1983-1987) at the mining company Ruhrkohle AG, Essen, at the mine Bergwerk Haus Aden, Bergkamen, Germany. Since 1988, he has been a Professor at the Jade University of Applied Sciences in Electrica Engineering, Electronic Devices, Mathematics. Since 1988, he has been an Evaluator of different bachelor and master study programs for the ASIIN. 1999-2004 - Dean of the Department of Engineering and Management FH OOW Wilhelmshaven. 2005-2007 -Head of the Institute of Seafaring in Leer. Address: Friedrich-Paffrath Street 101, 26389, Wilhelmshaven, Germany.

E-mail: jt@jade-hs.de

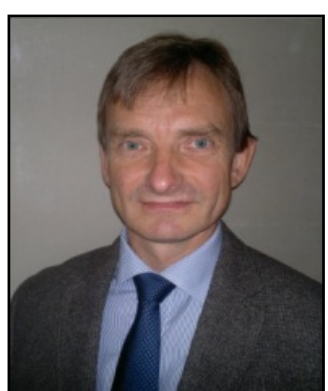

Andris Martinovs holds a degree of Doctor of Engineering Sciences and is an Associate Professor at Rezekne Higher Education Institution.

Education: in 2006 - Dr.sc.ing. in Mechanics and Strength of Material; in 1995 - Master' $\mathrm{s}$ Degree in Physics, Riga Technical University; in 1989 - Physicist, the University of Latvia.

Work experience: Rezekne Higher Education Institution - Lecturer (1993-2003), Assistant Professor (2003-2008), Associate Professor (since 2008), Director of professional higher education bachelor study programme "Mechatronics" (since 2007), Dean (acting) of the Faculty of Engineering (1996-2004). Environment Technology Centre, Member of the Board (since 2011).

His fields of research: exploring mechanical and electrical properties of materials, as elastomers, plastic, nano-structured coating, and development of prototypes in mechatronics.

Address: Atbrivosanas Alley 90, Rezekne, LV4601, Latvia.

E-mail: Andris.Martinovs@ru.1v 